\title{
Agricultura urbana en la ciudad de Valdivia, Chile. Un proceso de asentamiento
}

\author{
Urban agriculturel in the Valdivia city, Chile. A settlement process \\ Rodrigo P. Cabrera Verdezoto ${ }^{1}$, María Beatriz Vera Oyarzún ${ }^{2}$, \\ Rodrigo A. Echeverría Pezoa ${ }^{2}$, Carlos A. Amtmann Moyano ${ }^{3}$
}

\begin{abstract}
RESUMEN
El tema agrícola actualmente no solo involucra a los sectores rurales sino que también al sector urbano, debido a la migración rural/ urbano y a las características propias del desarrollo de la ciudad. En este contexto, la presente investigación tuvo como objetivo identificar los factores que han incidido en el proceso de asentamiento de las empresas agrícolas urbanas en la ciudad de Valdivia y caracterizar el perfil de estos empresarios. El universo de estudio estuvo constituido por las personas dedicadas a la agricultura urbana, con las siguientes características: que lo producido por los agricultores urbanos sea comercializado total o parcialmente de manera permanente y que se localicen en un radio no mayor a tres kilómetros del límite de la ciudad. La muestra estuvo constituida por 28 agricultores urbanos identificados mediante el sondeo "Snowball". El método utilizado fue el de la encuesta social y los datos obtenidos fueron sistematizados y luego procesados mediante estadística descriptiva. El factor que tiene mayor incidencia respecto del proceso de asentamiento de las empresas agrícolas urbanas es el abarcamiento de estos sectores que antes eran considerados rurales y en la actualidad están en los límites urbanos, con 53,57\%.

La edad promedio de los agricultores urbanos es de 54 años, el $61 \%$ de ellos son mujeres y el $57,14 \%$ cuentan con educación básica completa.
\end{abstract}

Palabras clave: agricultura urbana, proceso de asentamiento, migración rural/urbano.

\section{ABSTRACT}

The agricultural issue currently involves not only rural areas, but also the urban sector, due to rural / urban migration and the characteristics of the development of cities.The study was focused s on inhabitants of Valdivia city engaged in urban agriculture, with the following characteristics: urban farmers whose products are totally or partially sold permanently and occurring in the city within no more than three miles around. The sample consisted of 28 urban farmers identified by probing "Snowball". The method used was the social survey, and the data were systematized and then processed through descriptive statistics. The factor that has the greatest impact with respect to settlement process of urban agricultural enterprises is embracing these sectors once considered rural and is now within the city limits (3,57\% of the sample). The main results indicate that the average age of urban farmers is 54 years and that $61 \%$ of them are women, 57,14\% of urban farmers have completed basic education.

Key words: urban agriculture, settlement process, rural/urban migration.

\section{Introducción}

La agricultura ha sido siempre un tema ligado al medio rural, sin embargo, actualmente se realiza en zonas urbanas como consecuencia de las migraciones campo/ciudad, generada por bajos niveles de productividad y estancamiento de la actividad agrícola tradicional, subempleo o pobreza rural. Hasta 1930 existía en Chile más población rural que urbana, situación que posteriormente se revierte, aumentando de manera acelerada la población urbana $(86,65)$ y disminuyendo la población rural a13,4\% (Instituto Nacional de Estadísticas de Chile (INE), 2002). Lo anterior, responde principalmente a las migraciones campo-ciudad y a las políticas gubernamentales que en su gran mayoría apuntan a las grandes ciudades,

$1 \quad$ Facultad de Ciencias Agrarias, Escuela de Graduados, Universidad Austral de Chile, Independencia 641, Valdivia, Chile.

2 Instituto de Economía Agraria, Facultad de Ciencias Agrarias de la Universidad Austral de Chile, Independencia 641, Valdivia, Chile, fono: 56-63-221235. Email: bvera@uach.cl<mailto:bvera@uach.cl>

3 Facultad de Filosofía y Humanidades de la Universidad Austral de Chile Independencia 641, Valdivia, Chile.

Fecha de Recepción: 6 Mayo, 2013.

Fecha de Aceptación: 15 Agosto, 2013. 
beneficiando a la población urbana del país. De este modo, el proceso de urbanización se masificó y llegó a ser un proceso irreversible (Ramírez, 2011).

La política de industrialización impulsada desde el Estado bajo la idea de la sustitución de las importaciones y la aplicación de un modelo económico orientado hacia el mercado interno, son determinantes importantes del crecimiento y urbanización de las grandes ciudades. Las migraciones se orientan principalmente hacia los centros urbanos, donde existen mayores posibilidades y expectativas de empleo.

Otro factor que ha influido en el proceso de urbanización en Chile es la adopción de distintas formas de organización de la economía. Así, el modelo primario exportador permitió la generación de condiciones para el surgimiento de distintos poblados en las regiones centrales de Chile, regiones en donde los asentamientos, en distintos tamaños, logran cubrir y articular gran parte del territorio. Asimismo, los asentamientos en términos generales originan un impacto al medio ambiente que tiene que ver con la propia existencia de la población asentada en ciudades y localidades urbanas. Como se describe precedentemente, existe en el país un patrón de distribución con clara tendencia urbana y de marcada concentración (Universidad de Chile, 2000).

La Organización de las Naciones Unidas para la Alimentación y Agricultura (FAO) (2010) define a la agricultura urbana como toda la producción agrícola y ganadera que se realiza dentro de ciudades, pueblos y en sus zonas aledañas. Incluye desde pequeños huertos de hortalizas, producción de cultivos, frutales, plantas ornamentales, cría de ganado menor y vacas lecheras en la parte trasera de las viviendas. Gran parte de estos productos se destina al consumo propio y los excedentes se comercializan a nivel local. Por la ubicación geográfica y destino de consumo es una agricultura de bajos insumos, que no permite el uso con excesiva aplicación de agrotóxicos, con extrema economía en el uso del agua y cuidado de la fertilidad de sus tierras, manejo de los cultivos y de los animales. En un sentido amplio, ordenar el territorio implica optar entre alternativas de desarrollo, así como las relacionadas con el proceso de ordenamiento territorial que siempre presentarán criterios para decidir cuál uso resulta ser el más adecuado en función de los objetivos que ha definido la sociedad involucrada, lo que se manifiesta en un conjunto de políticas territoriales e instrumentos de planificación. Esto lleva a que todo proceso de ordenamiento territorial se desarrolle mediante tres fases de intervención: ordenamiento, necesidades y características de cada comuna, dando así las pautas de acción de planificación urbana, urbanización y construcción, entre otras normas de desarrollo urbano (Rovira, 2003).

Arenas (1999) sostiene que los Planes Reguladores Intercomunales tienen como principal objetivo el desarrollo físico de los territorios de las distintas comunas, cuyas relaciones generan influencias recíprocas sobre sus áreas urbanas y el entorno inmediato, por efecto de los servicios urbanos y las actividades productivas primarias, secundarias y terciarias que se realizan en cada comuna.

De acuerdo con lo anterior y debido a la escasez de información al respecto, esta investigación se planteó el objetivo de identificar los factores que han incidido en el proceso de asentamiento de las empresas agrícolas urbanas en la ciudad de Valdivia y caracterizar el perfil de estos empresarios.

\section{Materiales y Métodos}

\section{Área de Estudio}

El estudio se llevó a cabo en la ciudad de Valdivia de la comuna del mismo nombre, ubicada en la zona sur de Chile, capital de la Región de Los Ríos. Cuenta con una superficie de $951 \mathrm{~km}^{2}$ para una población de 140.559 habitantes, de los cuales $92,5 \%$ corresponde a población urbana y $7,5 \%$ a población rural (Chile, Instituto Nacional de Estadísticas (INE), 2002).

\section{Universo y muestra de estudio}

Se consideraron los siguientes criterios para determinar el universo de estudio:

- Que el total o una proporción de lo producido sea comercializado permanentemente.

- Que se produzca al interior de la ciudad o en los alrededores en un radio no superior a tres kilómetros (Vera y Zencovich, 2004).

La población estudiada estuvo constituida por todas las empresas agrícolas urbanas de la ciudad de Valdivia que satisfacen los criterios anteriormente indicados. 


\section{Metodología}

En abril de 2012 y a partir de la información de cuatro productores urbanos, nombres proporcionados por la Oficina del Programa de Desarrollo Social (PRODESAL) Valdivia, se fue constituyendo la muestra del estudio. Se utilizó la metodología "Snowball" o bola de nieve (Torres, 2005 y Bermejo, 2005), que consistió en localizar a los productores urbanos, los que condujeron a otros y así sucesivamente hasta por saturación, la muestra de estudio quedó constituida por 28 productores urbanos, ubicados en 13 localidades de la ciudad de Valdivia.

El instrumento utilizado para identificar los factores que han incidido en el proceso de asentamiento de estos productores y determinar el perfil del empresario agrícola urbano fue un cuestionario semiabierto o semiestructurado.

\section{Resultados y Discusión}

\section{Proceso de asentamiento de las empresas agrícolas urbanas}

En cuanto a la ubicación de las empresas agrícolas urbanas, se pudo determinar que el $39,29 \%$ se ubica dentro de área urbana y el 60,71\% está ubicada en el perímetro urbano según el plano regulador vigente de la ciudad de Valdivia, Región de Los Ríos. Sin embargo, los resultados de Pantanalli (2009) indican que el 42,9\% de las empresas agrícolas urbanas se encuentran en la ciudad, mientras que el $57,1 \%$ restante está ubicado en el perímetro urbano.
En relación con la procedencia de los agricultores urbanos del estudio, se determinó que el mayor porcentaje de los agricultores urbanos $(53,57 \%)$ ha permanecido siempre en el mismo lugar (Figura 1). Estos espacios que eran rurales hoy se encuentran dentro de los límites urbanos de la ciudad, debido al crecimiento industrial y poblacional de la ciudad de Valdivia. El 17,86\% de los productores agrícolas urbanos son migrantes de sectores rurales, hacia la ciudad de Valdivia, asimismo el 14,29\% son consecuencia de las migraciones entre ciudades, manteniendo el mismo comportamiento los productores que siempre han permanecido en la ciudad de Valdivia. Lo que menciona Bianchetti (2004) tiene similitud con los resultados de esta investigación, quien sostiene que el actual crecimiento de muchas de las ciudades intermedias en Chile ha originado la creación de nuevas áreas urbanas. Este es el caso de la ciudad de Quillota, en Chile, donde hace varios años se encuentra en un proceso de transformación urbana; proceso que involucra la creación de un parque industrial en el sector oriente de la ciudad, situación que ha llevado a los planificadores locales a ampliar los límites urbanos de la ciudad incorporando a esta área, que históricamente se consideró rural. Sin embargo, Sepúlveda (2004) sostiene que a nivel latinoamericano se ha producido un aumento en las poblaciones de las ciudades, básicamente producto de una importante migración producida desde los sectores rurales. Este dato se contradice con los obtenidos en esta investigación.

Respecto de la calidad de vida, se observó que el $42,86 \%$ de los agricultores encuestados considera que

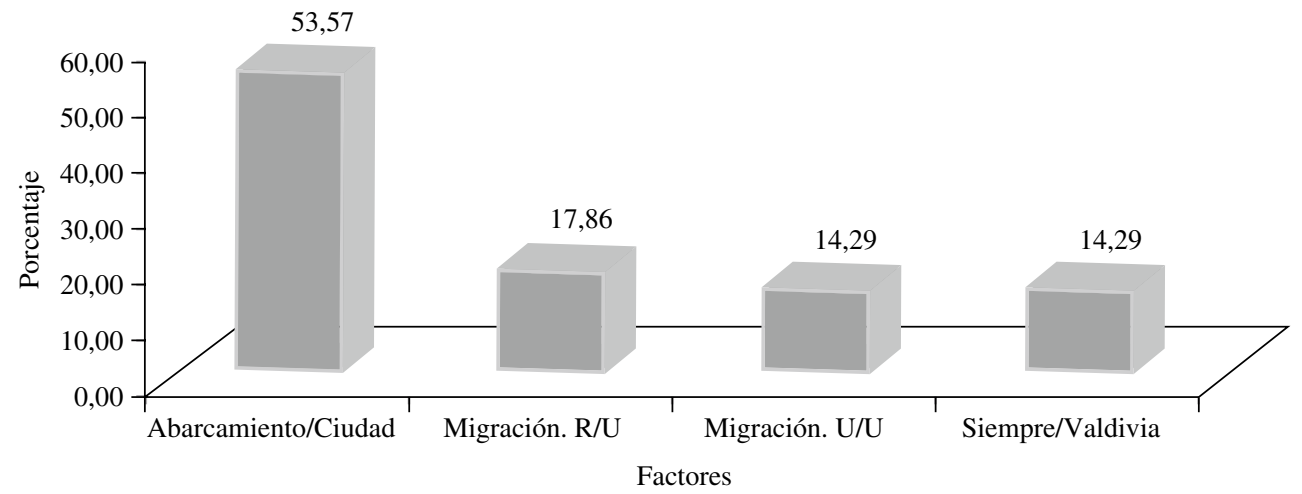

Figura 1. Procedencia de los agricultores urbanos en Valdivia. 
su calidad de vida ha mejorado significativamente en relación con los servicios básicos como agua potable, luz, teléfono fijo, recolección de basura e internet. El $28,57 \%$ indica que en la actualidad las autoridades están más comprometidas con sus sectores y han mejorado las redes viales pavimentándolas. Lo anterior ha permitido que la locomoción llegue hasta su lugar de trabajo, permitiéndoles acceder con más facilidad hasta el centro de la ciudad. El $21,43 \%$ considera que todo sigue igual a los años anteriores y $7,14 \%$ se encuentra en la categoría otros, quienes indican que se han implementado espacios recreacionales como parques y recintos deportivos (Figura 2). Al respecto, Max Neef y col. (1986) señalan que el mejor proceso de desarrollo será aquel que permita elevar más la calidad de vida o condiciones de vida de las personas y que en tal sentido corresponde especificar lo que determina la calidad de vida de las personas. Al igual que Araujo (1982), quien considera que los componentes del bienestar humano podrían ser los siguientes: acceso a la educación y salud, sistemas de igualdad de oportunidades, movilidad social tanto vertical como horizontal, uso y control de los recursos productivos; y participación en la redistribución del ingreso, en el reparto de excedentes, en la inversión, en el consumo y en el ejercicio del poder.

El tiempo de permanencia en la actividad agrícola urbana de estos empresarios está concentrada principalmente en el rango mayor a los 16 años (71\%), con un tiempo promedio de 28 años, cifras que se asemejan a lo indicado por Cardemil (2004), quien indica que el 54,3\% de los empresarios agrícolas del sector sur de la provincia de Valdivia permanecen en esta actividad entre 10 y 29 años, con un promedio de 23,4 años. Por otra parte, Vera y Moreira (2009) sostienen que las microempresas agrícolas de la provincia de Valdivia tienen en promedio 7,2 años de funcionamiento, con un rango entre los 2 y 53 años.

La mitad de los agricultores encuestados se dedica a esta actividad por tradición familiar (Cuadro 1), y el 71,43\% de los agricultores urbanos de la ciudad de Valdivia son de origen rural. Los datos concuerdan con lo señalado por Leal (2006), quien sostiene que la mayoría de los empresarios agrícolas del sector norte de la provincia de Valdivia se inician en este rubro principalmente por tradición familiar, representando $59 \%$.

En relación con la superficie utilizada, se puede indicar que los 28 agricultores urbanos del estudio en la ciudad de Valdivia presentan una superficie utilizada de 80,79 hectáreas. Se observó que el $42,86 \%$ de los agricultores urbanos cuentan con superficies entre 1 y 5 hectáreas, seguido de los agricultores que no superan 1 hectárea de superficie $(35,71 \%)$. Al respecto, Pardo (2008) manifiesta que

Cuadro 1. Razones para dedicarse a la agricultura urbana

\begin{tabular}{lrr}
\hline & \multicolumn{2}{c}{ Razón agricultores urbanos } \\
\cline { 2 - 3 } & $\left(\mathrm{N}^{\circ}\right)$ & $(\%)$ \\
\hline Tradición familiar & 14 & 50,00 \\
Mejorar economía & 10 & 35,72 \\
Hobby & 2 & 7,14 \\
Relaciones personales & 1 & 3,57 \\
Iniciativa propia & 1 & 3,57 \\
Total & 28 & 100,00 \\
\hline
\end{tabular}

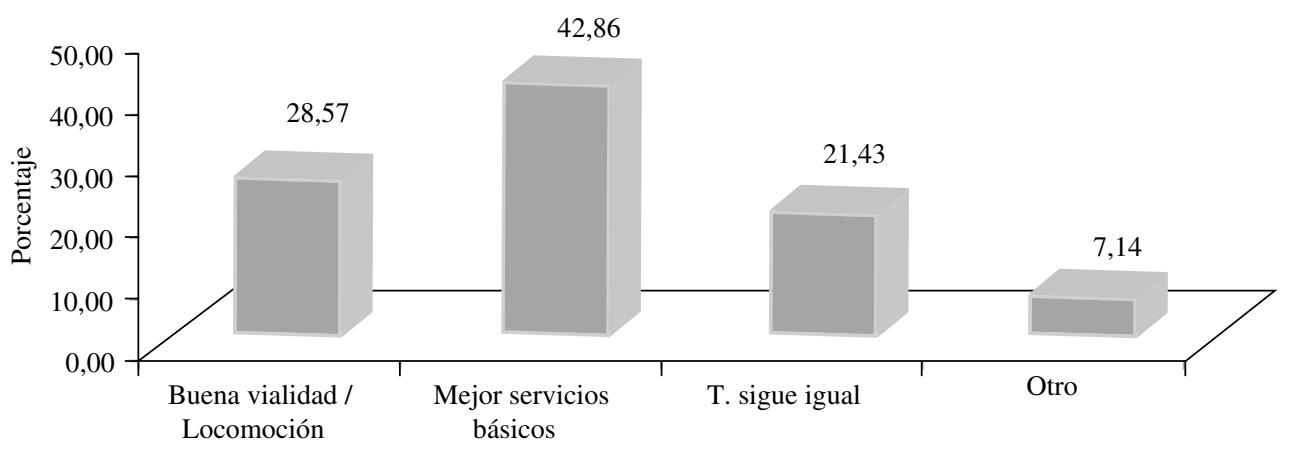

Aspectos

Figura 2. Aspectos de calidad de vida. 
18 agricultores de la provincia de Ranco, Región de Los Ríos, que representa el 35,29\% de ese estudio, no superan una hectárea de superficie para ejercer la agricultura urbana, seguido de los agricultores que producen en un rango de 1 a 10 hectáreas con $27,45 \%$, contradiciéndose a los resultados de esta investigación.

En cuanto a las perspectivas de los empresarios agrícolas urbanos de la ciudad de Valdivia, en su mayoría piensa aumentar su infraestructura, sin embargo, hay un porcentaje $(17,86 \%)$ que no tiene ningún tipo de perspectivas, debido a su avanzada edad. Lo anterior, coincide con lo que señala Vera y Zencovich (2004), Leal (2006) y Cardemil (2004), quienes indican que las principales expectativas de los empresarios agrícolas urbanos es mejorar y aumentar la infraestructura.

\section{Perfil del Empresario Agrícola Urbano de Valdivia}

Caracterización social y experiencia en la agricultura urbana. El análisis permitió determinar que existe una mayor proporción de mujeres $(61 \%)$ dedicadas a la agricultura urbana en relación con los hombres (39\%). Al respecto Sánchez y col. (2008), en un estudio realizado en la provincia de Valdivia, determinaron que el 56,5\% son mujeres que se dedican a la agricultura urbana, cifra similar al presente estudio. La actividad de la agricultura urbana permite a las mujeres realizar tareas cotidianas y producir sus propios alimentos para el consumo del hogar, dejando los excedentes para la comercialización, contribuyendo así a la economía familiar.

En cuanto a la edad hay diferencias entre los empresarios agrícolas urbanos de la ciudad de Valdivia, las que fluctúan entre los 30 y 75 años, con un promedio de 54 años (Cuadro 2), dato que coincide con lo mencionado por Sánchez y col. (2008), quienes señalan que la edad promedio de los empresarios urbanos de la provincia de Valdivia es de 53 años. En esta investigación se observa una distribución de edad heterogénea, concentrada entre los 41 y 50 años $(32,14 \%)$, valor que corrobora la autora Pantanalli (2009).

Mellado (1995) indica que los niveles de educación entre los empresarios agrícolas es diversa y que la tendencia es hacia tener más educación, estudios técnicos y universitarios. Sin embargo, los datos determinados en esta investigación no reflejan
Cuadro 2. Distribución de los agricultores urbanos, según edad.

\begin{tabular}{lcc}
\hline \multicolumn{3}{c}{ Dedicación agricultores urbanos } \\
\hline \multirow{2}{*}{$\begin{array}{c}\text { Edad } \\
\text { (años })\end{array}$} & \multicolumn{2}{c}{ Agricultores urbanos } \\
\hline $30-40$ & 4 & $(\%)$ \\
$41-50$ & 9 & 14,29 \\
$51-60$ & 5 & 32,14 \\
$61-70$ & 7 & 17,86 \\
$>70$ & 3 & 25,00 \\
& & 10,71 \\
Total & 28 & 100,00 \\
\hline
\end{tabular}

lo indicado por dicho autor, ya que el $57,14 \%$ cuenta tan solo con estudios básicos completos (Figura 3).

Asimismo, se observó que el $75 \%$ de los agricultores urbanos no tiene profesión, situación que en varios casos los motivó a dedicarse a la agricultura urbana, pensando en una alternativa como fuente de ingreso.

La mayoría de los empresarios son de origen rural $(71,43 \%)$ y están directamente relacionados con el área agrícola, dedicando tiempo completo a la agricultura urbana, por ser esta la única fuente laboral y que por su origen y experiencia manejan (Figura 1). Al respecto Mujica (2001) señala que en la mayoría de las ocasiones las personas pasan de trabajadores dependientes a empresarios, por un problema social y que muchas veces están relacionados con la falta de oportunidades laborales o simplemente por una necesidad personal de independencia económica, con el riesgo que ello conlleva.

En relación con la actividad adicional que dedican estos agricultores urbanos, $68 \%$ no efectúa actividad adicional, mientras $32 \%$ tiene otra actividad distinta a la agricultura urbana, lo que podría deberse a que la mayoría de los agricultores son mujeres y destinan el tiempo a las actividades domésticas. Al respecto Cardemil (2004) sostiene que 56,6\% de los agricultores del sector sur de la provincia de Valdivia ${ }^{4}$ no realizan otra actividad diferente a la agricultura, mientras que el $43,4 \%$ realiza actividades tales como dueña de casa, comerciante, maestro constructor y otras actividades de menor frecuencia.

Existen agricultores urbanos de la ciudad de Valdivia que solo viven de esta actividad,

$4 \quad$ Sector sur de la provincia de Valdivia, actualmente provincia de Lago Ranco. 


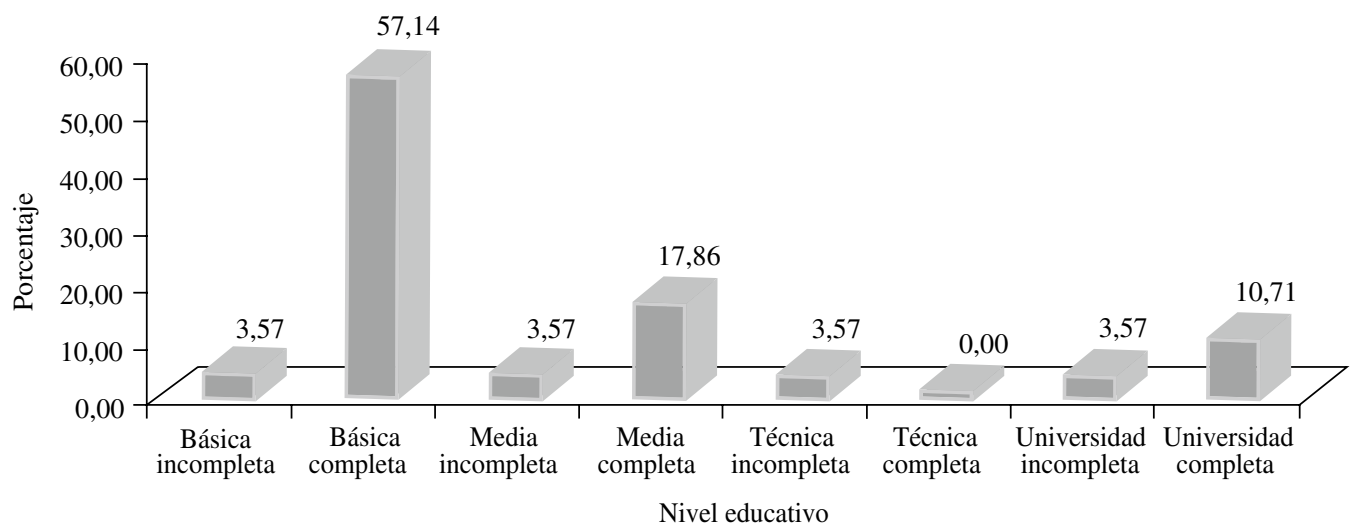

Figura 3. Nivel de educación de los agricultores urbanos.

demostrando que es una fuente de ingreso importante y que sirve para sustentar gastos de alimentación y la educación de sus hijos. El 85,71\% de ellos vive de esta actividad y solo el $14,29 \%$ indica no vivir de ella.

Vera y Zencovich (2004) sostienen que en la agricultura tradicional se trabaja todos los días de la semana, ya que en el mundo rural no existen distracciones que saquen del trabajo a los agricultores; en cambio en la agricultura urbana este fenómeno no se manifiesta de igual manera, debido a que existen innumerables distracciones y actividades complementarias que se puedan realizar.

En esta investigación el rubro más representativo, en relación con la dedicación de los agricultores urbanos, es el de las hortalizas $(39,29 \%)$, seguido del cultivo de los frutales $(21,43 \%)$, y $17,86 \%$ de los agricultores encuestados se dedican al rubro de las plantas ornamentales. Los resultados antes mencionados tienen gran similitud con lo señalado por Pardo (2008), quien indica en el estudio realizado en la provincia de Ranco sobre agricultura urbana que el rubro de las hortalizas es el principal, con $39,20 \%$. Sin embargo, Vera y Zencovich (2004) y Pantanalli (2009) en sus estudios mencionan que el principal rubro de los agricultores de Valdivia es el de las plantas ornamentales, con $42 \%$ y $57,1 \%$, respectivamente, seguido de las hortalizas con $32 \%$ y $21,4 \%$, respectivamente.

En cuanto al tiempo destinado a la agricultura urbana, el 64,29\% dedica los 7 días de la semana a realizar esta actividad, $14,29 \%$ destina de 3 a 6 días, mientras que solo $7,14 \%$ dedica entre 1 y 2 días para su actividad (Cuadro 3), coincidiendo con los datos del estudio realizado por Sánchez y
Cuadro 3. Tiempo destinado a la agricultura urbana

\begin{tabular}{lcc}
\hline \multicolumn{3}{c}{ Dedicación agricultores urbanos } \\
\hline Días & $\mathrm{N}^{\mathbf{0}}$ & $\%$ \\
\hline $1-2$ & 2 & 7,14 \\
$3-4$ & 3 & 14,29 \\
$5-6$ & 5 & 14,29 \\
7 & 18 & 64,29 \\
\hline Total & 28 & 100,00 \\
\hline
\end{tabular}

R/U: Rural/Urbano, U/U: Urbano/Urbano.

col. (2008). Sin embargo, es importante mencionar que el porcentaje de agricultores que no dedica los 7 días de la semana a la agricultura urbana es porque realizan actividades adicionales, razón por la que más del $35 \%$ destina menos días de trabajo a esta actividad.

\section{Conclusiones}

El asentamiento de las empresas agrícolas urbanas en la ciudad de Valdivia se debe principalmente a un proceso de abarcamiento (crecimiento) de la ciudad, a consecuencia de las modificaciones del plano regulador de la ciudad, así como a la emigración del campo a la ciudad.

El $60,71 \%$ de las empresas agrícolas urbanas están ubicadas en la periferia o límite urbano de la ciudad de Valdivia, debido al crecimiento industrial y poblacional.

Alrededor del $43 \%$ de los agricultores urbanos considera que ha mejorado significativamente su calidad de vida al estar ubicados dentro del radio 
urbano de la ciudad, debido que cuentan con mayores y mejores servicios básicos, como agua potable, luz, teléfono fijo, recolección de basura e internet.

Aumentar la infraestructura es la principal proyección señalada por los empresarios agrícolas urbanos, porque les permite mejorar su productividad $\mathrm{y}$ aumentar los ingresos.

El $71 \%$ de los empresarios agrícolas urbanos tiene un tiempo de permanencia en la actividad igual o mayor a 16 años y la ejerce por tradición familiar, porque le permite una mejoría económica y se dedica principalmente al cultivo de hortalizas.

La edad promedio de los empresarios agrícolas urbanos es de 54 años y el $61 \%$ de ellos son mujeres. El 10,71\% tiene estudios universitarios completos, situación que los ha llevado a dedicarse a la agricultura urbana como una forma de hacer negocio. En su mayoría no realiza actividades adicionales y dedica los 7 días de la semana a esta actividad.

\section{Literatura Citada}

Araujo, J.

1982. Agenda para el futuro. Balance y perspectivas del desarrollo Rural en América Latina y el Caribe, una visión desde el IICA. Serie: Desarrollo Institucional $N^{\circ} 13$. San José. Costa Rica. IICA, 382 p.

Arenas, F.

1999. Organización territorial y desarrollo regional en Chile. En Estudios Geográficos, Consejo Superior de Investigaciones Científicas, Instituto de Economía y Geografía, Madrid, Tomo LX, 234, pp. 101-119.

Bermejo, B.

2005. Planificar una investigación. Muestreo. Disponible (en línea) http://www2.uiah.fi/projects/metodi/252.htm. Consultado: 19/02/2012.

Bianchetti, A.

2004. Transformaciones espaciales, construcción de identidades urbanas poblacionales y movilización del capital social comunitario en nuevos emplazamientos urbanos de la ciudad de Quillota: el caso de las Villas Agustín Avezón y Los Aromos. Tesis Licenciado Antropología, Universidad Austral de Chile. Santiago, Chile, 96 p.

Cardemil, M.

2004. Caracterización de la micro y pequeñas empresas agrícolas del sector Sur de la provincia de Valdivia, Chile. Tesis Licenciado en Agronomía. Universidad Austral de Chile. Valdivia, Chile, 166 p.

Chile, Instituto Nacional de Estadísticas (INE).

2002. Censo de Población y Vivienda. www.ine.cl/cd2002/ sintesiscensal.pdf. Consultado: 11/01/2012.

Leal, R.

2006. Caracterización de la micro y pequeña empresa agrícola del sector Norte de la Provincia de Valdivia, Chile. Tesis Licenciado en Agronomia. Universidad Austral de Chile. Valdivia, Chile, $118 \mathrm{p}$.

Max Neef, M., Elizalde, A. y Hopenhayn, M. 1986. Desarrollo a escala humana: una opción para el futuro. Development Dialogue. $N^{\circ}$ Especial. Fundación Dag Hammarsk jöld, $25 \mathrm{p}$.

Mellado, E.

1995. Experiencias de capacitación empresarial en la IV Región. El área urbana. In Minkner - Bunger, M. (eds). Micro y pequeña empresa: buscando nuevos espacios para su desarrollo. Fundación Naumann, Chile, pp. 208-210.
Mujica, J.

2001. El inicio de la empresa familiar y sus fundadores. Mundo PYMES. La revista de la pequeña y mediana empresa, Chile. 2: 25-27.

Organización de las Naciones Unidas para la Alimentación y Agricultura (FAO).

2010. Perspectivas económicas y sociales. La lucha contra el hambre y la pobreza. http://www.fao.org/docrep/012/ al377s/al377s00.pdf. Consultado: 29/01/2012.

Pantanalli, K.

2009. Análisis comercial de las empresas agrícolas urbanas de la ciudad de Valdivia. Tesis Licenciado en Agronomía. Universidad Austral de Chile. Valdivia, Chile, 93 p.

Pardo, S.

2008. Caracterización socioeconómica y comercial de la agricultura urbana de la provincia de Ranco, Región de Los Ríos. Tesis Licenciado en Agronomía. Universidad Austral de Chile. Valdivia, Chile, $97 \mathrm{p}$.

Ramírez, M.

2011. Territorio y sistema de ciudades. El medio urbano y el medio rural. Liceo Polivalente Juan Antonio Ríos. http://www. fileden.com/files/2011/10/11/3207726/My\%20Documents/ Gu\%C3\%ADa\%203\%C2\%B0\%20Ciudad\%20Cont.\%20 N\%C2\%B03.pdf. Consultado: 01/09/2012.

Rovira, A.

2003. Bases Para el Ordenamiento Territorial Ambientalmente Sustentable de la Región. Marco Legal del Ordenamiento Territorial Santiago de Chile. Gobierno Regional Región Metropolitana. http:www.gobiernosantiago.cl/medios/ Archivos_Otas/MA R_LEGAL_OT. doc. Consultado: 30/03/2012.

Sánchez, C., Vera, B., y Bohm, L. 2008. Perfil del empresario agrícola urbano de la provincia de Valdivia, Región de Los Ríos. Agro Sur (Chile), volumen 36 (2): 101-110.

Sepúlveda, A.

2004. Asentamientos urbanos marginales: El caso del campamento Llancahue, Tesis Licenciado Antropología. Universidad Austral de Chile. Valdivia Chile. 125 p.

Torres, M.

2005. Cuadernos estancias de investigación. La identidad profesional docente del profesor de educación básica en México. http www.crefal.edu.mx/bibliotecadijital/CEDEAL/ acervo_digital/coleccion_crefal/cuadernos_estancias/ cuadernos_estancias.htm. Consultado: 24/02/2012. 


\section{Universidad de Chile}

2000. Informe país, estado del medio ambiente de Chile. LOM Ediciones. Chile, pp. 327-364.

\section{Vera, B. y Zencovich, J.}

2004. Agricultura urbana en la ciudad de Valdivia, Chile. Una nueva alternativa microempresarial. Agro Sur (Chile), volumen 32 (2): 71-78.
Vera, B. y Moreira, V.

2009. Caracterización de la microempresa agrícola del sur de Chile. Idesia (Chile). Universidad de Tarapacá, Facultad de Ciencias Agronómicas, Arica, Chile. Volumen 27, $\mathrm{N}^{\circ} 3$, pp. 89-99. 